\title{
Prediction of Lunar Reconnaissance Orbiter Reaction Wheel Assembly Angular Momentum Using Regression Analysis
}

\author{
Russell DeHart \\ Systems Engineer, NASA Goddard Space Flight Center, Space Science Mission Operations, \\ KBRwyle
}


- Study Motivation \& Goal

- Lunar Reconnaissance Orbiter (LRO) Overview

- Spacecraft

- Angular momentum control

- Methodology

- Removing effects of maneuvers

- Forming piece-wise regression model

- Results

- Test against 2013 - 2014 telemetry

- Conclusion \& Future Work 


\section{Introduction}

Study Motivation \& Goal

LRO Overview

LRO Angular Momentum 


\section{Study Motivation \& Goal}

- Motivation

- Conserve fuel

- Fuel remaining at start of 2016: $\approx 34 \mathrm{~kg}$

- Current fuel usage: $\approx 4 \mathrm{~kg} / \mathrm{yr}$

- Thrusters used for momentum unloads $(\Delta \mathrm{Hs})$

- Includes station keeping \& $\Delta \mathrm{Hs}$, but not orbit changes

- Increase science collection

- Three instruments safed during $\triangle H s$ : DLRE, LEND, LOLA

- One also decontaminated for 24 hrs thereafter: LAMP

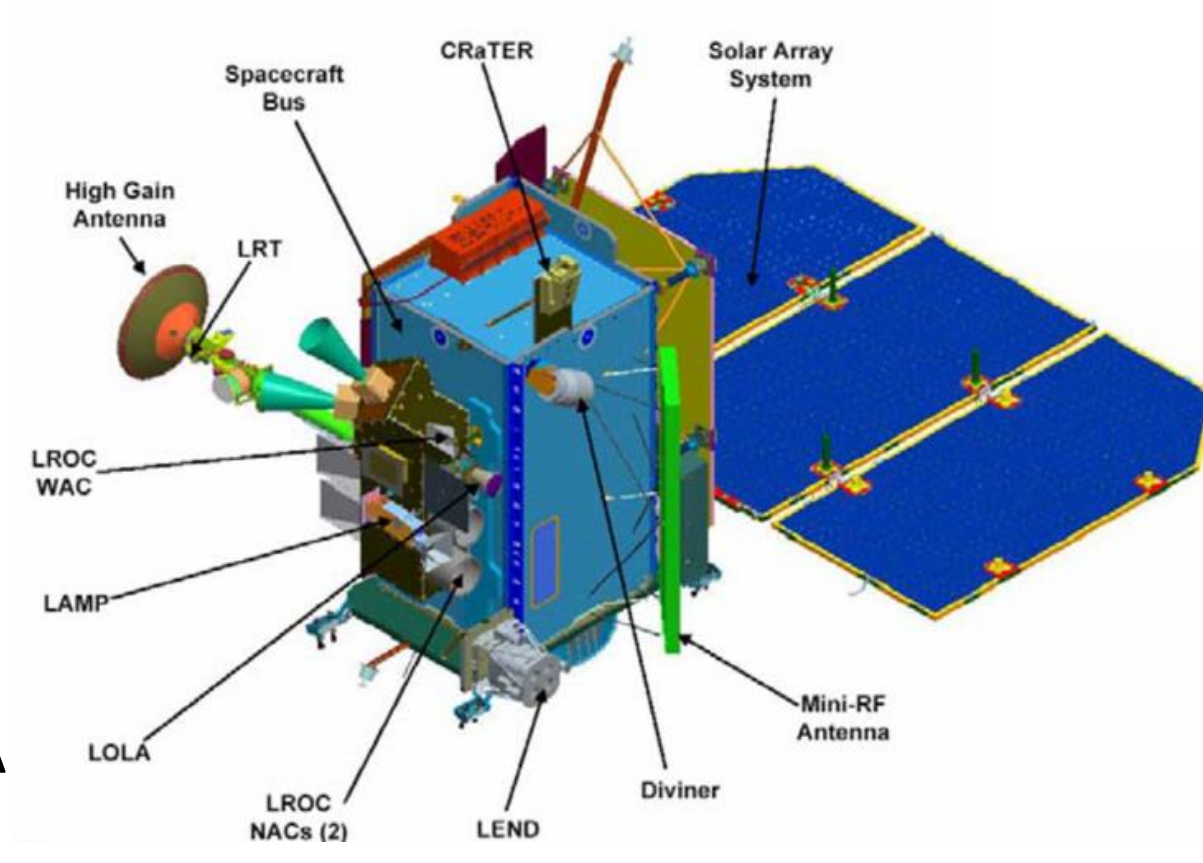

- Legacy planning procedures often resulted in unloads being performed more frequently than needed

GOAL:

Build model to accurately predict growth in LRO angular momentum, enabling optimal scheduling 


\section{LRO Spacecraft Overview}

- Orbit

- Changed several times, now $20 \mathrm{~km} \times 150 \mathrm{~km}$

- Nominally nadir-pointing

- Solar Array (SA)

- Has inner \& outer gimbals

- Uses tracking and parked configurations

- Tracking

- $|\beta|<30^{\circ}$ : Gimbals track sun, but with $+30^{\circ}$ outer gimbal offset

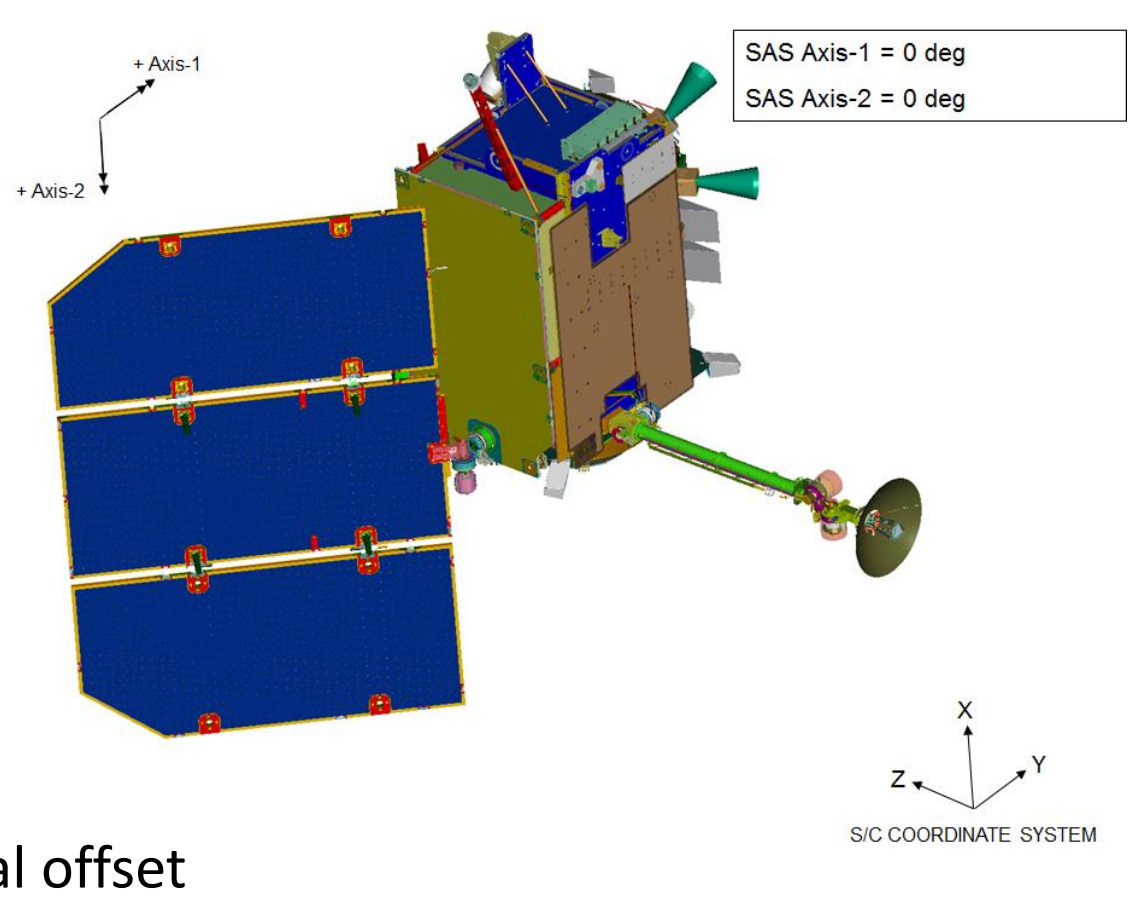

- $30^{\circ}<|\beta|<35^{\circ}$ : Gimbals track sun, but with $-30^{\circ}$ outer gimbal offset

- Parked

- $35^{\circ}<|\beta|<55^{\circ}$ : park inner gimbal at $-90^{\circ}$, outer gimbal at $+45^{\circ}$

- $|\beta|>55^{\circ}$ : park inner gimbal at $-90^{\circ}$, outer gimbal at $+15^{\circ}$

- Offsets and fixed angles selected to maintain gimbals $<45^{\circ} \mathrm{C}$ 


\section{LRO Attitude \& Disturbance Torques}

- Attitude control

- Reaction wheel assembly (RWA) used to:

- Slew spacecraft

- Maintain attitude, countering disturbance torques

- RWA angular momentum capacity

- $115 \mathrm{Nms}$ at nominal bus voltage

- $80 \mathrm{Nms}$ at minimum bus voltage

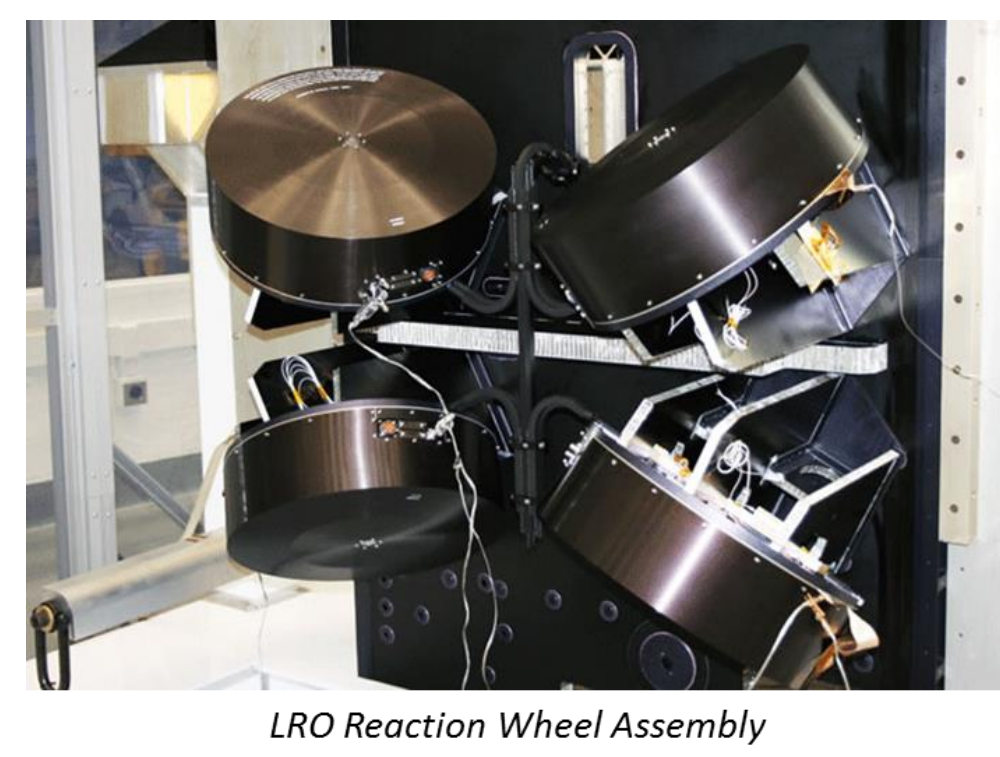

- Since lunar orbit, only 2 significant disturbance torques

- Gravity gradient torque

- Movement of solar array dominates changes in LRO inertia matrix

- Solar radiation pressure (SRP) torque

- Solar array dominant term due to large moment arm
All disturbance torque orbitaveraged values are simply functions of $\beta$ 


\section{LRO Angular Momentum}

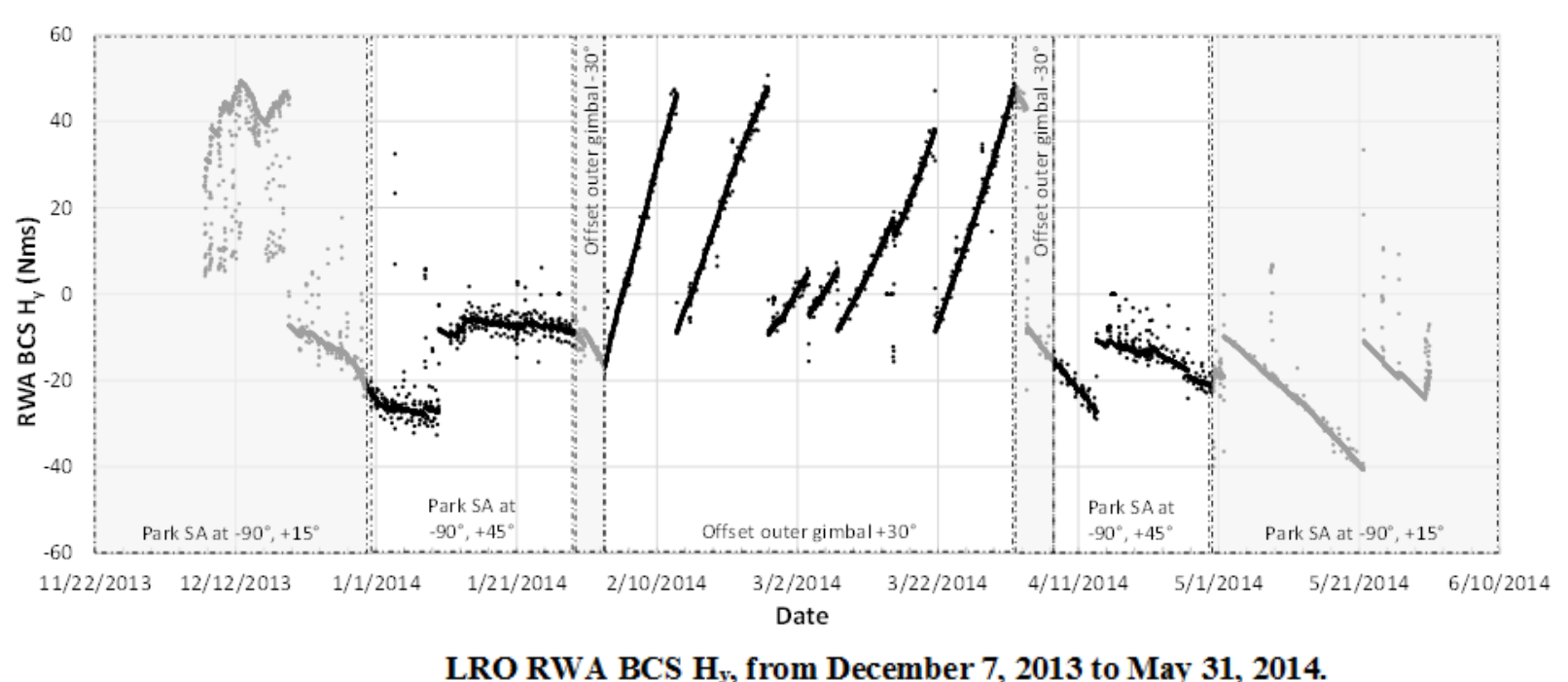

LRO RWA BCS Hy, from December 7, 2013 to May 31, 2014.

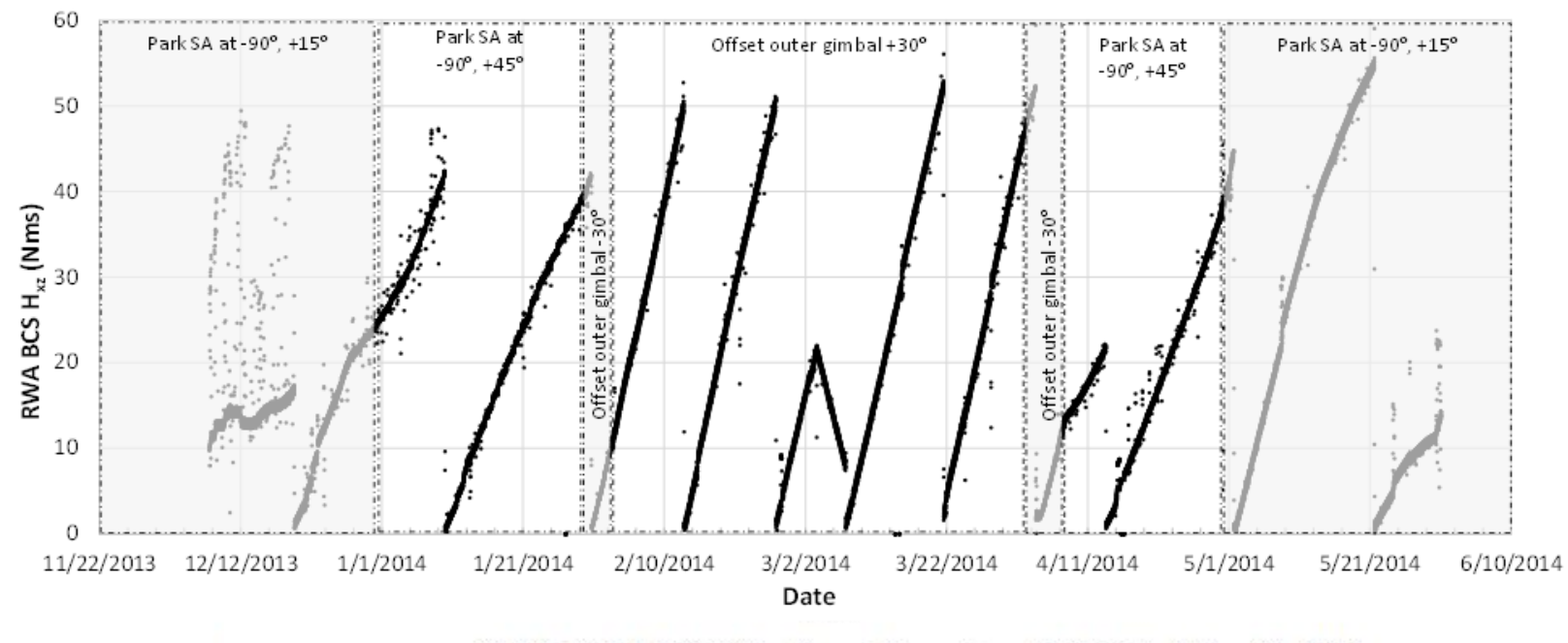

LRO RWA BCS Hz, from December 7, 2013 to May 31, 2014.

- Body Coordinate System (BCS) $\mathrm{H}_{\mathrm{y}}$ very dependent on solar array configuration (and hence $\beta$ )

- Activities, like pitch \& yaw campaigns in Dec 2013 can overwhelm trend

\section{Model must include both regression against $\beta$ and adjustments for activities}




\section{Methodology}

Overview

Determining effects of maneuvers

Forming piece-wise regression model 


\section{Methodology Overview}

- Piece-wise polynomial least-squares regression

- One year's worth of data

- Regress Hy and Hxz against beta separately, with Htot simply being RSS

- October 2014 - October 2015

- Covers full cycle of beta values

- Linear and cubic regressions

- Define piece boundaries based on SA modes

- First remove effects of slews \& unloads to see 'natural' trend

- Analyze angular momentum in vicinity of each and every activity

- Collect perturbations induced by activities \& make ruleset

- Perform regression

- Use regression coefficients - and perturbations - to predict future momentum 


\section{Determining Effects of Maneuvers}

- Perform linear regressions on either side of activity

- Perturbance is vertical step change

- Remove step change to smooth data

- Repeat several hundred times

- Example: 0.5-Nms increase in Hy caused by $59^{\circ}$ LROC roll

- Make "ruleset"

- Group slews by type and beta regime at time of slew

- Define changes in angular momentum due to slew/beta pairs
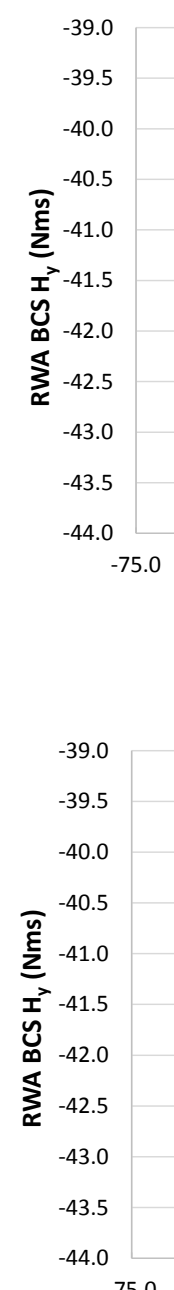

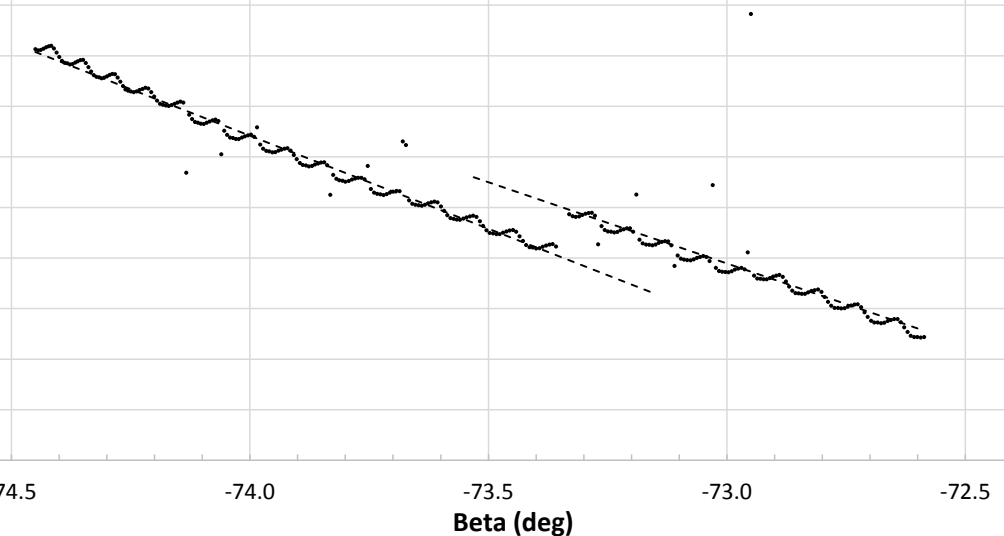

LRO RWA BCS Hy, from June 16, 2014 to June 17, 2014, with linear regressions.

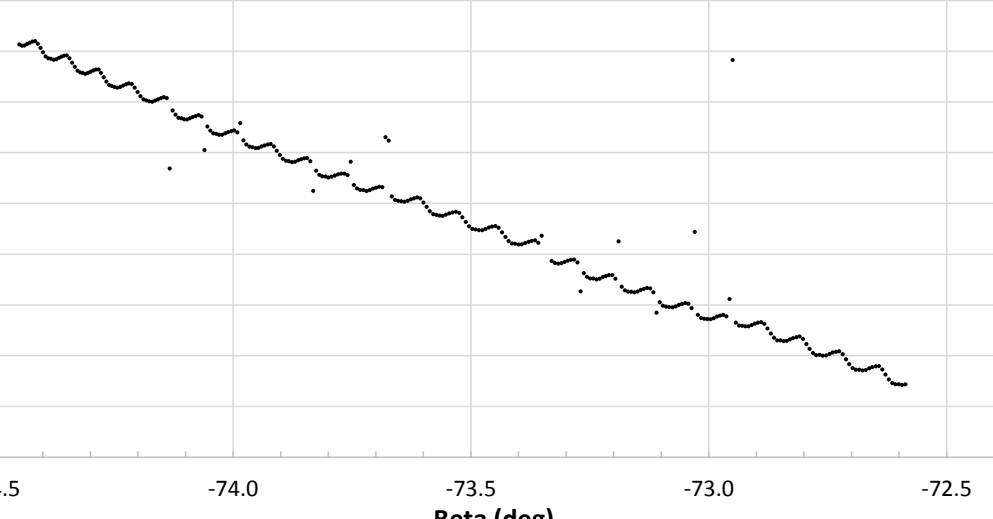




\section{Forming Piece-Wise Regression Model}

- Perform regressions with now smooth data

- Least squares

- On Hy \& Hxz

- SA modes guide boundary selection

- Example at right: beta going from $30^{\circ}$ to $0^{\circ}$

- Record six decimal places for coefficients for sufficient precision in angular momentum

- $\mathrm{R}^{2}$ values $>0.99$ typical

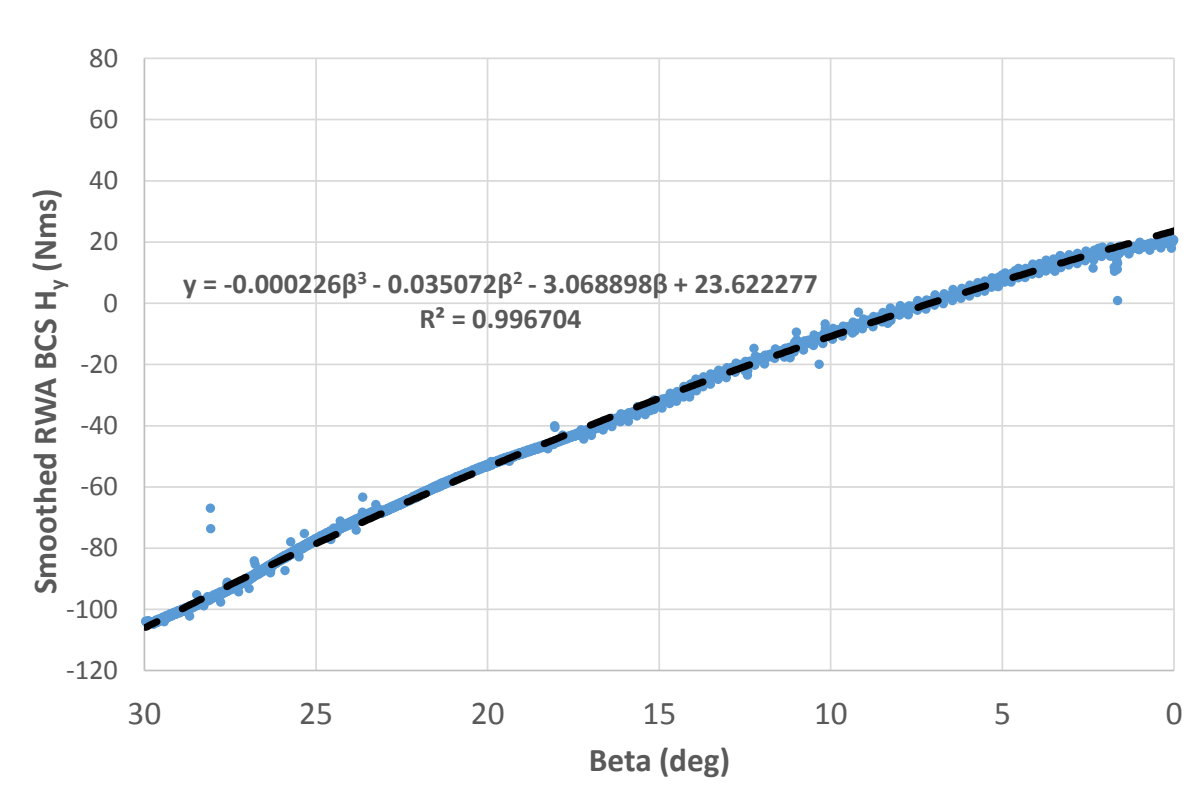

Smoothed LRO RWA BCS $\mathrm{H}_{\mathrm{y}}$, for $\beta$ spanning from $30^{\circ}$ to $0^{\circ}$.

\section{Selected linear regression when} strongly linear, arbitrarily selected cubic otherwise-it's all about simply achieving a good fit 


\section{Results}

Hy

$\mathrm{H} x \mathrm{z}$

Total H 


\section{3 - 2014 Test: Hy}

- Use regression from Oct 2014 Oct 2015 to predict angular momentum in previous year

- Immediately after each unload

- Initialize model to values from telemetry (Hy $\approx-9 \mathrm{Nms}, \mathrm{Hxz} \approx 0 \mathrm{Nms}$ )

- Predict angular momentum until next unload

- 'Baseline' prediction based on:

- Regression coefficients

- Beta values predicted at time by FD

- Add step changes using list of

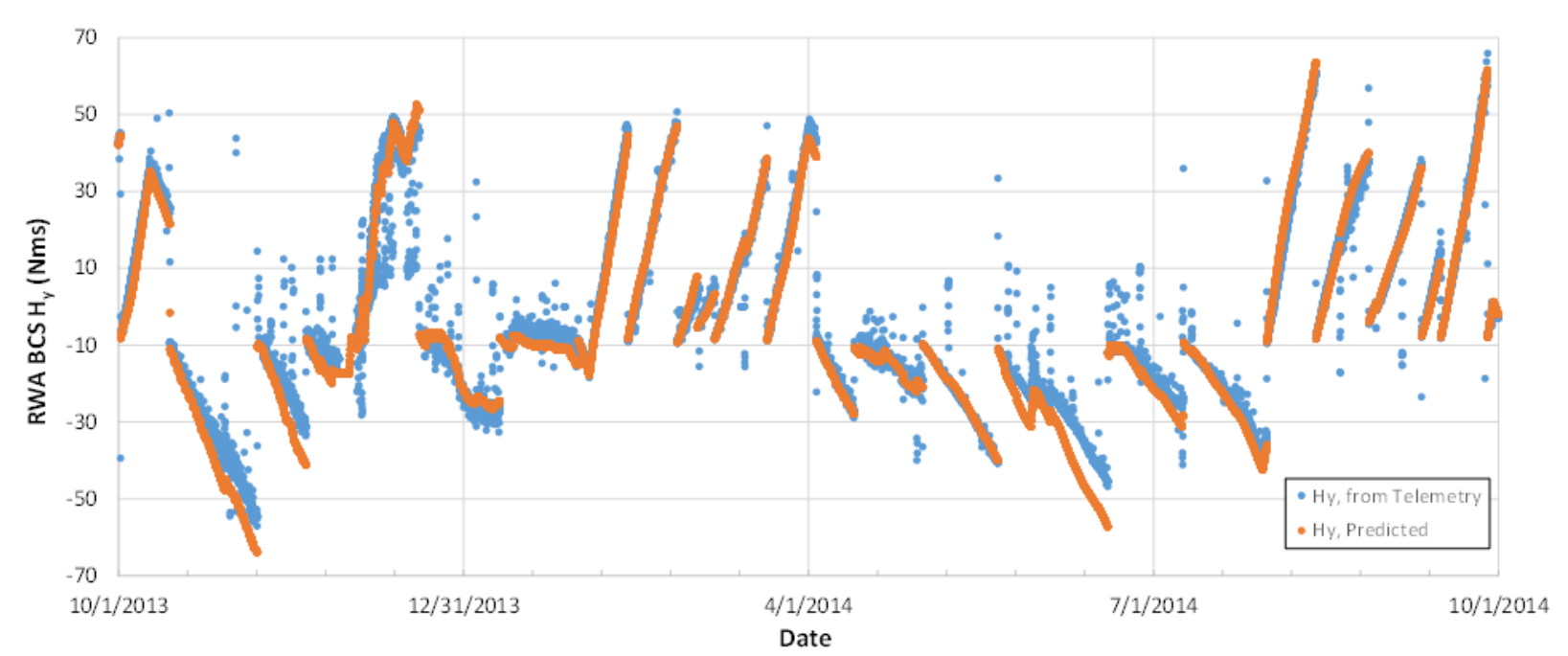
performed slews 


\section{3 - 2014 Test: Hxz}

- Largely very good agreement except for Dec 2013 \& June 2014

- Corresponds to when beta has just retreated away from $\pm 90^{\circ}$

- Morphology of predicted trend not matching observations

- Max prediction errors approaching $10 \mathrm{Nms}$

- Have since formed new regression equations with better, though not perfect fit

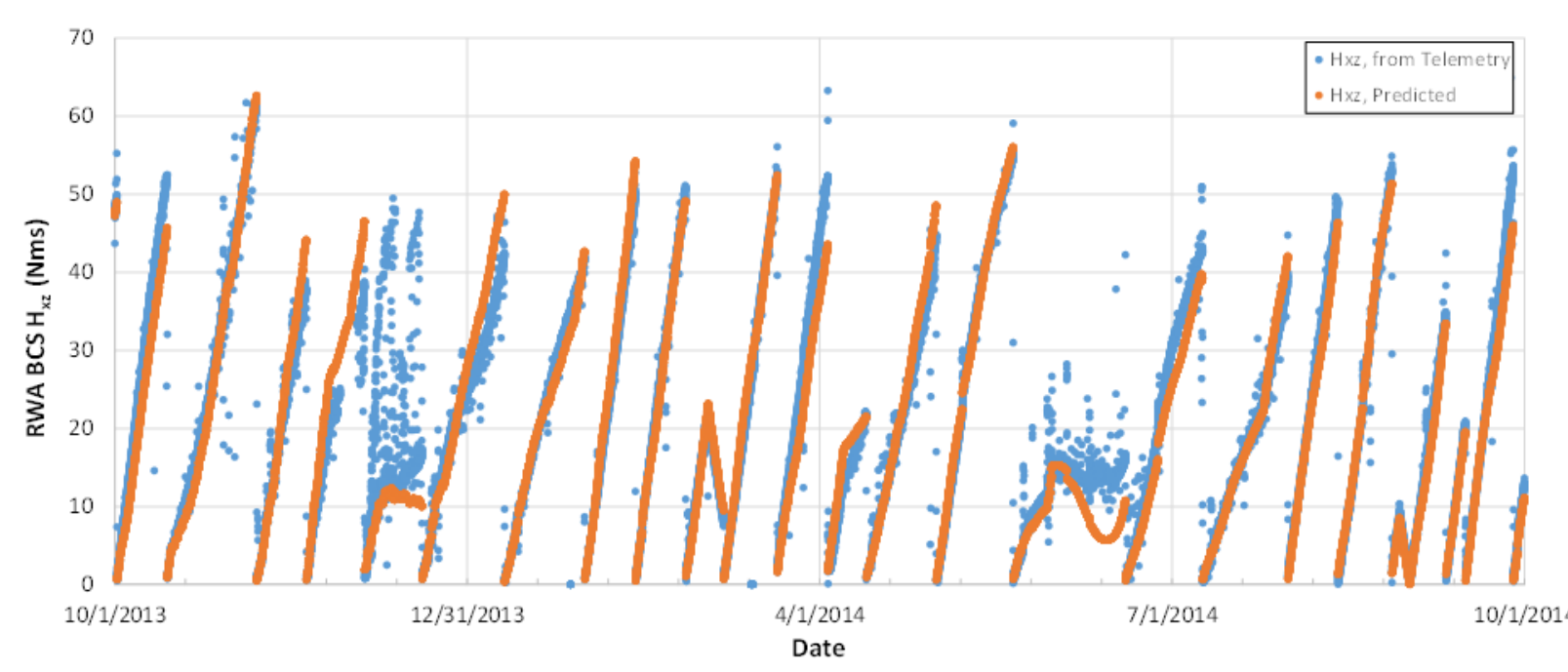

- Total $\mathrm{H}$ is most important

Achieved good fit, though most

challenging period is when beta has just retreated away from $\pm 90^{\circ}$ 


\section{3 - 2014 Test: Total H}

- Total H used for unload planning

- Small prediction errors at time of next unload:

- Mean = 3.7 Nms

- Median = 2.7 Nms

- $\operatorname{Max}=10.6 \mathrm{Nms}$

- Operational results would likely be even better than here

- OPSCON would include using tool intermittently

- Opportunities for re-synching model

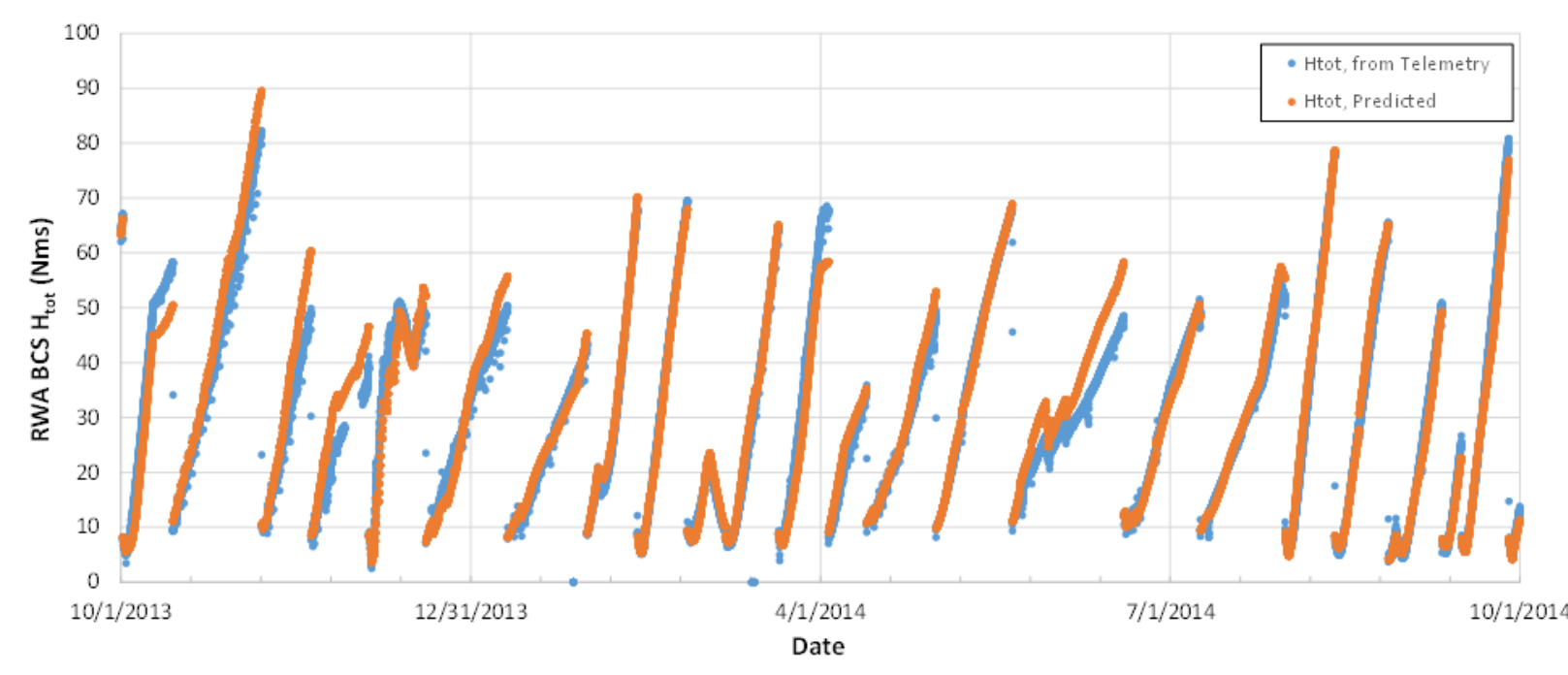
against telemetry 


\section{Conclusion}

Summary

Future Work

Discussion 


\section{Summary}

- LRO operations now uses these accurate angular momentum predictions

- Especially accurate if schedule known far in advance

- Currently schedule 1-2 weeks in advance

- Mission investigating scheduling farther out based on results of study

- Momentum unloads now performed more optimally

- Momentum target now selected depending on trend experienced after unload

- For example, don't select $-10 \mathrm{Nms}$ if trend is to become more negative

- Delays next unload by $3-9$ days, depending on trend

- Have waited as much as 42 days between unloads

- Study has enabled other improvements

- Identified slews that counteract trend in angular momentum

- Hope to eventually control angular momentum without using propellant 


\section{Future Work}

- Developing tool to deliver to operations team

- Analyzing angular momentum behavior as beta retreats from $\pm 90^{\circ}$

- Testing the use of select operational slews to decrease angular momentum

- Off-nadir attitude providing beneficial gravity-gradient torque

- SRP smaller factor 


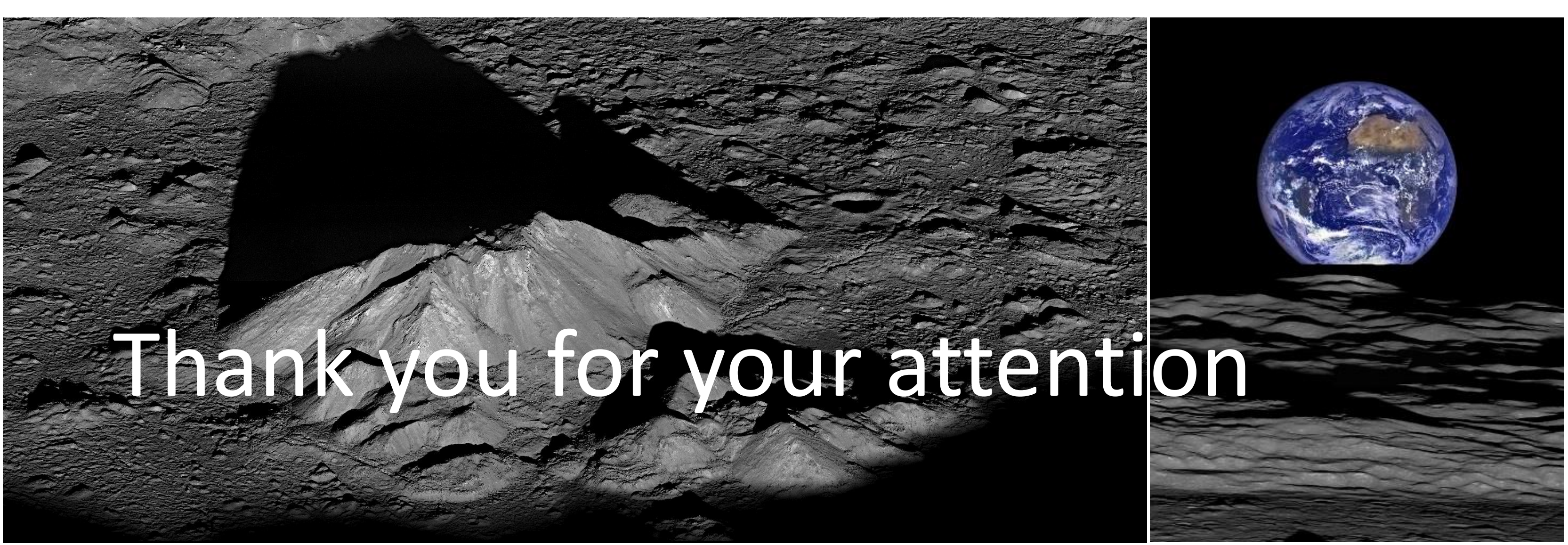

